\title{
FOOTPRINTING STUDIES OF THE INTERACTION OF QUINOMYCIN ANTIBIOTIC UK63052 WITH DNA: COMPARISON WITH ECHINOMYCIN
}

\author{
KeITH R. Fox \\ Dept. Physiology \& Pharmacology, University of Southampton, \\ Bassett Crescent East, Southampton SO9 3TU, UK
}

(Received for publication March 22, 1990)

\begin{abstract}
The interaction between UK63052, a novel derivative of the quinomycin group of bifunctional intercalating antibiotics, with DNA has been investigated by footprinting techniques and the results compared with echinomycin. UK63052 binds strongly but reversibly to DNA and decreases the gel mobility of most DNA fragments, although the mobility of bent kinetoplast DNA is increased. The drug binds selectively to the dinucleotide $\mathrm{CpG}$ though not all such sequences present good binding sites. Binding is best when CG is surrounded by AT base pairs. UK63052 and echinomycin have different effects on DNA structure as assessed by changes in the sensitivity to modification by diethylpyrocarbonate. The results are interpreted by suggesting that substitutions on the chromophores affect the precise details of DNA recognition.
\end{abstract}

The quinoxaline antibiotics are cyclic octadepsipeptides to which two quinoxaline chromophores are attached and are produced by various strains of Streptomyces. The group has been divided into two classes, the triostins and the quinomycins, according to the nature of the cross-bridge which is a simple disulfide for the former and a thioacetal for the latter ${ }^{1,2)}$. The best studied compound in this series is echinomycin (Fig. 1). Various naturally occurring derivatives have been isolated and differ only in the amino acid at the $N$-methyl valine position ${ }^{3)}$.

These compounds owe their antitumour and antibiotic activities to the ability to bind to DNA which they do by the mechanism of bifunctional intercalation ${ }^{4,5)}$. All the natural derivatives bind best to $\mathrm{GC}$-rich DNAs ${ }^{6,7)}$ and footprinting studies have shown that the binding site consists of the dinucleotide $\mathrm{CpG}^{8 \sim 10)}$. A number of semisynthetic analogues have been produced bearing different chromophores. Substitution of quinoline residues resulted in derivatives with subtly different DNA binding isotherms ${ }^{1.1)}$, in particular an increased affinity for poly (dA-dT), though DNAase I footprinting revealed no difference in selectivity ${ }^{12)}$. In contrast, addition of an amino-group to the 3-position of the quinoxaline ring produced a compound which retained the selectivity for $\mathrm{CpG}$ yet did not bind to all such sites, appearing to recognise sites of the type $(\mathrm{A} / \mathrm{T}) \mathrm{CG}(\mathrm{A} / \mathrm{T})^{12)}$.

Other related antibiotics such as the luzopeptins, which possess a cyclic decadepsipeptide, have also been isolated ${ }^{13)}$ and display a very different sequence specificity ${ }^{14,15)}$. More recently a new quinomycin antibiotic UK63052 (Fig. 1) has been characterised and shown to contain two 3-hydroxy quinoline chromophores attached to a cyclic octadepsipeptide ${ }^{16}$. The cyclic peptide differs from echinomycin by having an unusual cyclic amino acid in place of valine and in the substituents on the cross-bridge.

In this paper we have examined the binding of UK63052 to DNA by DNAase I footprinting on several DNA fragments. 
Fig. 1. Structures of echinomycin and UK63052.

(A) Echinomycin, (B) UK63052.

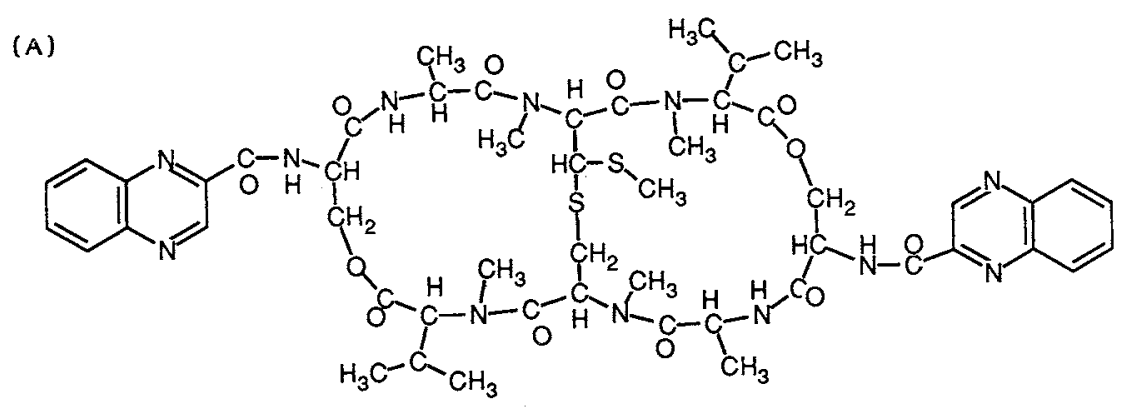

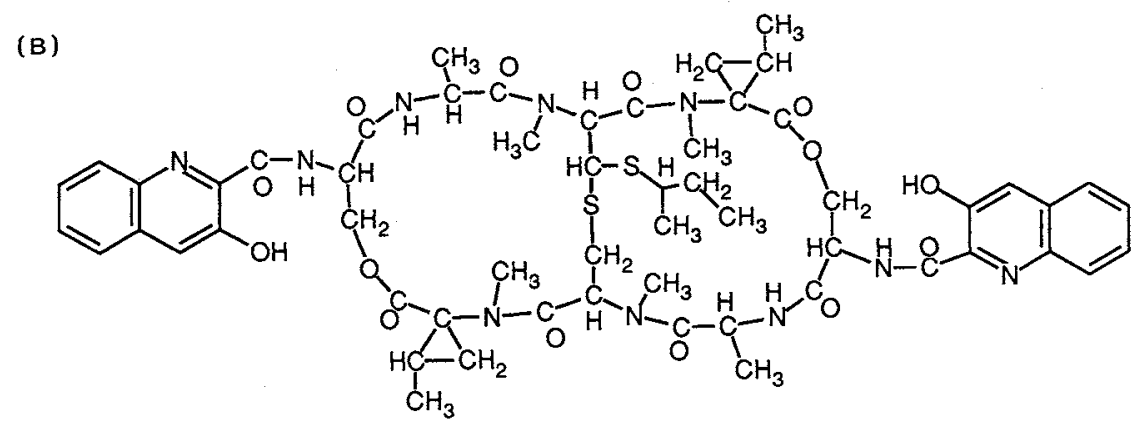

\section{Materials and Methods}

\section{Drugs}

UK63052 was a gift from Pfizer Central Research, Sandwich, UK, echinomycin was obtained from Dr. M. J. WARING, University of Cambridge. Since both drugs are known to be insoluble in aqueous buffers stock solutions (1 $\mathrm{mm}$ ) were prepared in dimethyl sulfoxide and diluted to working concentrations in $10 \mathrm{~mm}$ Tris $-\mathrm{HCl} \mathrm{pH} 8.0$ containing $10 \mathrm{~mm} \mathrm{NaCl}$ immediately before use. Some antibiotic precipitates from solution during this dilution so that the concentrations quoted are only approximate.

\section{DNA Fragments}

The 160 base pair tyr $\mathrm{T}^{17)}$ and 270 base pair kinetoplast ${ }^{18)}$ DNA fragments were isolated and labelled at their $3^{\prime}$-ends using reverse transcriptase and either $\alpha-{ }^{32} \mathrm{P}$-dATP or $\alpha-{ }^{32} \mathrm{P}$-dCTP as previously described $^{19,20)}$; their sequences are shown in Fig. 2. The 270 base pair DNA fragment designated B5 was obtained from Mr. W. M. Brown, Dept. Physiology \& Pharmacology, University of Southampton and contains $47 \% \mathrm{G}+\mathrm{C}$ residues.

\section{Footprinting}

DNAase I footprinting and diethylpyrocarbonate (DEPC) modification reactions were performed as previously described ${ }^{19,21)}$. The products of digestion were resolved on $6 \sim 8 \%$ polyacrylamide gels containing $8 \mathrm{M}$ urea run for about 2 hours at 1,500 V. After electrophoresis gels were fixed in $10 \%$ acetic acid, dried under vacuum at $80^{\circ} \mathrm{C}$ and subjected to autoradiography at $-70^{\circ} \mathrm{C}$ using an intensifying screen.

\section{Results}

Echinomycin has been shown to have no effect on the mobility of DNA on non-denaturing 
Fig. 2. Sequences of the tyrT and kinetoplast DNA fragments.

The bases bearing the radioactive label are underlined.

TyrT DNA

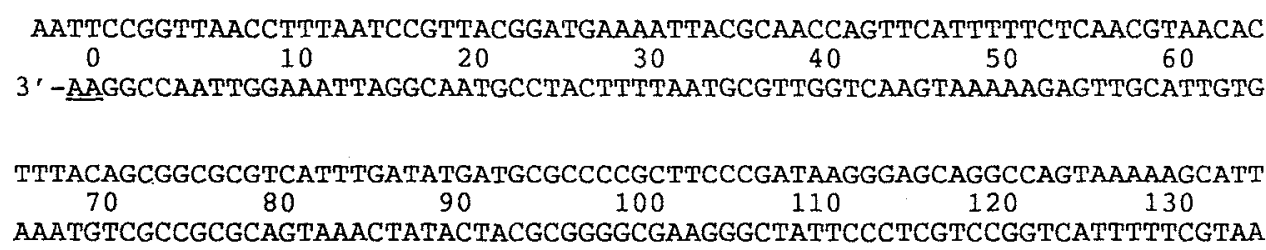

ACCCCGTGGTGGGGGTTCCC

$140 \quad 150$

TGGGGCACCACCCCCAAGGGCT-5'

Kinetoplast DNA

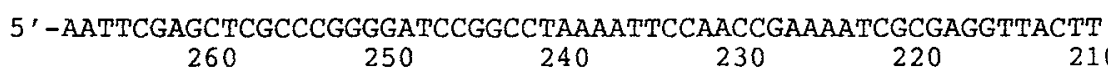

AAGCTCGAGCGGGGCCCTAGGCCGGATTTTAAGGTTGGCTTTTAGCGCTCCAATGTT

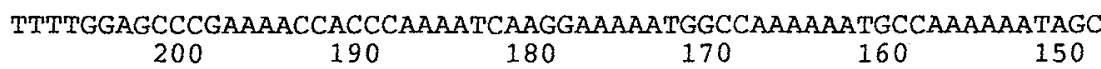

AAAACCTCGGGCTTTTGGTGGGTTPTAGTTCCTTTTTACCGGTTTTTTACGGTTTTTTATCG

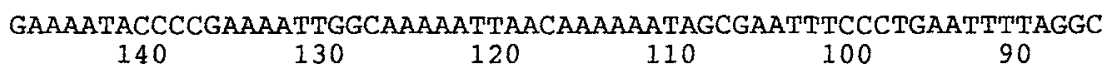

CTMTTACGGGGCTTTTAACCGTITTTAATTGTTTTTTATCGCTTAAAGGGACTTAAAATCCG

GAAAAAACCCCCGAAAATGGCCAAAAACGCACTGAAAATCAAAATCTGAACGTCTCCGGATC

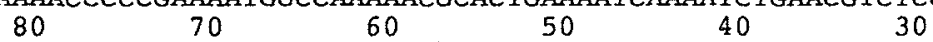

CTPTTTTGGGGGCTTTPACCGGTTTTTGCGTGACTTTTAGTTTTAGACTIGCAGAGGCCTAG

CTCTAGAGTCGACCTGCAGCCCCAA-3

2010

GAGATCTCAGCTGGACGTCGGGGTTCGA

polyacrylamide gels ${ }^{14)}$, presumably because the drug dissociates during electrophoresis. In contrast luzopeptin causes a marked reduction in DNA mobility ${ }^{14)}$, an effect caused by tighter binding, possibly some form of covalent attachment. Before studying the sequence selective binding of UK63052 to DNA we have therefore investigated its effect on the mobility of DNA in non-denaturing gels. The results of such experiments are presented in Fig. 3.

Concentrations of $30 \mu \mathrm{M}$ UK63052 and above reduce the mobility of tyrT DNA in contrast to echinomycin which has no effect. The "smiling" of the echinomycin treated sample is presumably due to diffusion of UK63052 within the gel matrix and is not seen with echinomycin-treated samples alone. With kinetoplast DNA, which adopts a highly bent structure on account of its phased runs of adenines has an abnormally slow rate of gel migration, UK63052 causes an increase in mobility. This effect is peculiar to kinetoplast DNA since fragment B5 which is a similar length and base composition shows the usual decrease in mobility. It appears that UK63052 is able to remove the bending found in kinetoplast DNA.

\section{Sequence Selectivity}

Fig. 4 presents the results of DNAase I digestion of tyrT DNA, labelled on either strand, in the presence and absence of UK63052 and echinomycin. The cleavage pattern in the drug treated lanes is very 
different from that in the controls; the results for echinomycin are similar to those previously reported $^{9)}$. On inspection it is evident that the two antibiotics produce similar but not identical protections. Looking first at the labelled bottom strand echinomycin protects from cleavage around all the CpG sites ${ }^{9}$, which are located at positions $35,58,73,76,78,95,100$ and 107 . In contrast UK63052 only produces clear blockages around positions 58 and 100 , with weaker protections at $70 \sim 80$ and no protection around position 35 . The blockages around 75 and 100 , both of which contain more than one $\mathrm{CpG}$ binding site, extend over shorter regions for UK63052 than echinomycin. The pattern of enhancements is also different for the two ligands; witness the relative band intensities around position 65 . With the labelled top strand both ligands protect from cleavage at all the $\mathrm{CpG}$ sites, though positions 100 and 58 are protected at lower drug concentrations. The pattern of enhancements around $65 \sim 70$ is again different.

It has recently been shown that echinomycin does not produce DNAase I footprints at all available CpG sites on the kinetoplast DNA fragment ${ }^{20)}$. Fig. 5 presents DNAase I footprints of UK63052 on this DNA. For the labelled top strand clear protections can be seen for both ligands around positions 55, 70, 135 and 200, each of which represents the dinucleotide step CpG. Similar sequences at positions 85 and 106 are unaffected while that at position 147 is protected better by UK63052 than echinomycin. The same protections can be seen with the label on the bottom strand, again notice that 147 is better protected by UK63052 than echinomycin. The patterns of enhancement on this DNA fragment are similar for both compounds.

\section{Effects on Local DNA Structure}

The results described above reveal that, as well as possessing slightly different sequence recognition properties, echinomycin and UK63052 produce different enhancements in DNAase I activity. If these enhancements are attributed to drug-induced alterations in DNA structure then the two ligands must be affecting local DNA structure to different extents. One way of showing this in greater detail is the sensitivity to DEPC modification. It has been demonstrated that echinomycin renders certain adenines hyper-reactive to DEPC ${ }^{20 \sim 24)}$, especially those distal to echinomycin binding $\operatorname{sites}^{20)}$. This has been interpreted as arising from local unwinding of the DNA helix. Fig. 6 presents the results of DEPC modification of tyrT and kinetoplast DNA in the presence and absence of UK63052 and echinomycin. In the presence of both ligands certain adenines are rendered hyper-reactive. The results for echinomycin are similar to those previously reported ${ }^{20,21)}$, those for UK63052 are similar but not identical. For tyrT DNA labelled on the bottom strand (Fig. 6D) echinomycin induces major enhancements at positions 79 and 83/84, with UK63052 
Fig. 4. DNAase I footprinting of tyrT DNA labelled at the $3^{\prime}$-end of either strand in the absence (CON) and presence of UK63052 and echinomycin (ECHY).
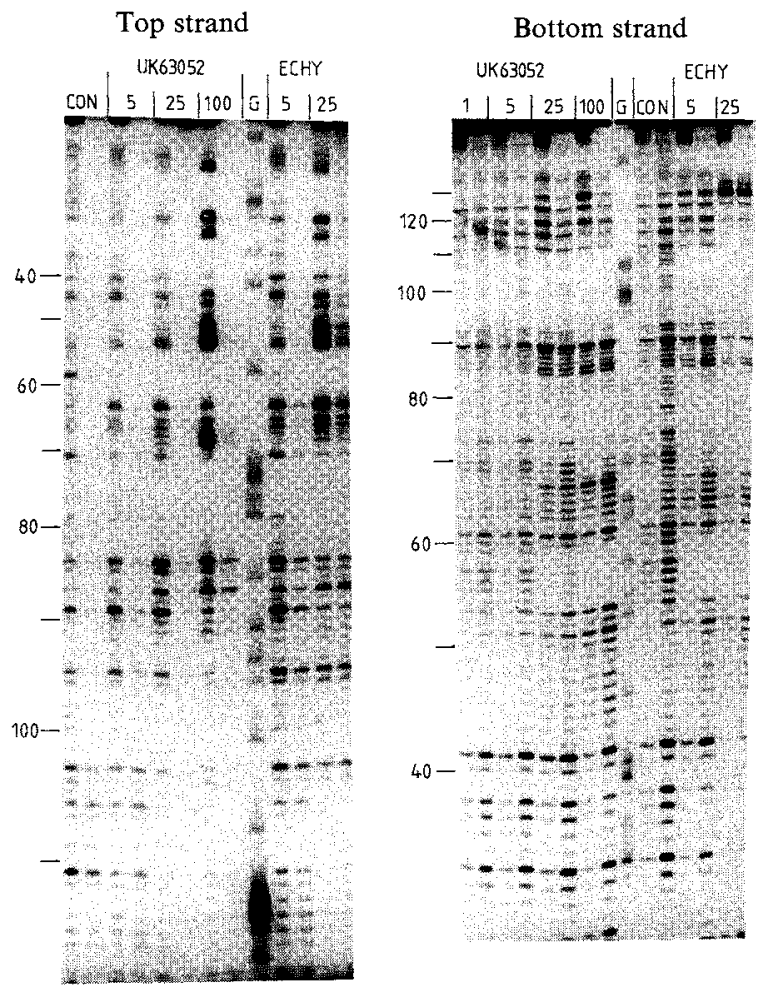

Drug concentrations $(\mu \mathrm{M})$ are indicated at the top of the gel lanes. Each pair of lanes corresponds to digestion by the enzyme for 1 and 5 minutes. The numbers correspond to the sequence shown in Fig. 2. The track labelled "G" is a dimethylsulfate-piperidine marker specific for guanine.

the enhancement at position 79 is much weaker and an additional strong band corresponding to modification of adenine 89 is apparent. With tyrT top strand (Fig. 6C) UK63052 induces novel enhancements at positions 82,71 and 64 while those corresponding to positions $109,87,57 / 56$, which are strongly enhanced by echinomycin, are much weaker; those at 92,47 and 42 are common to both ligands. For the kinetoplast top strand (Fig. 6B) all the strong echinomycin induced enhancements are at adenines in the sequence CGA; most of these are visible in the pesence of UK63052 along with new products at positions 62 and 118. Few enhancements are seen on the kinetoplast bottom strand (Fig. 6A), since this contains few sequences of the type CGA, however UK63052 produces novel cleavage products at positions 172 and 129. The reasons for these differences in DEPC modification are not clear but they serve to confirm that the DNA structural changes induced by the two ligands are not identical.

\section{Discussion}

The results presented in this paper demonstrate that UK63052 binds to DNA and that its sequence selectivity is similar to that of echinomycin. It does however differ from echinomycin in the details of its binding; some sites are less well protected and the DNA structural changes induced are not identical. Each of these aspects will be discussed in turn below.

The ability of UK63052 to increase the mobility of kinetoplast DNA is unusual since most drugs and 
Fig. 5. DNAase I footprinting of the kinetoplast DNA fragment labelled on either strand in the absence (CON) and presence of UK63052 and echinomycin (ECHY).

Bottom strand

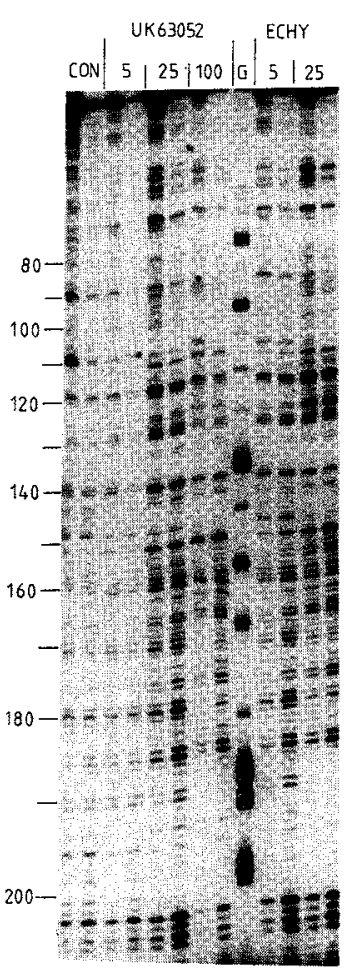

Top strand

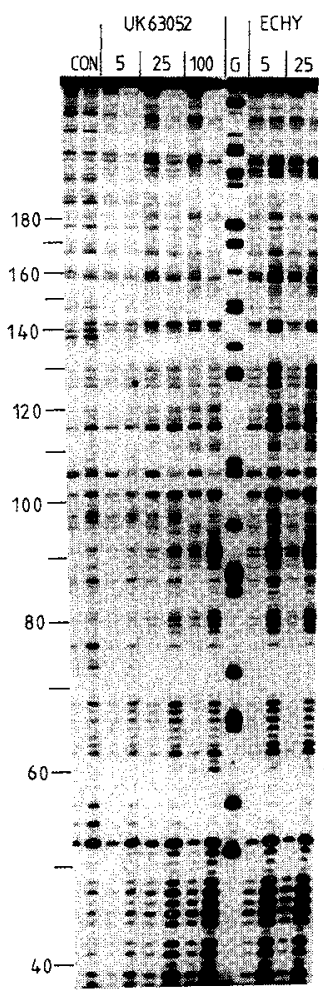

Other details are as for Fig. 4.

proteins reduce DNA mobility. A simlar effect has previously been shown for distamycin, but was only achieved by including the ligand in the gel matrix and running buffer. On the basis of DNAase I footprinting it was suggested that echinomycin removed the bending caused by phased runs of adenines ${ }^{20}$. If we assume that the ligand is bound only to the $\mathrm{CpG}$ sites then a maximum of $15 \mathrm{drug}$ molecules can be bound per duplex. Of these 5 sites are outside the putative bending region and one more can be discounted due to the site overlap at 219/221. It seems then that no more than 9 drug molecules are responsible for the increase in mobility of the DNA, i.e. about one ligand for every two helical turns. It has been suggested that luzopeptin affects DNA mobility by covalent attachment to the $\mathrm{DNA}^{14)}$. Is this a possibility for UK63052? The evidence suggests not. Luzopeptin produces its effects at very low concentrations (nanomolar) ${ }^{25)}$ whereas under similar conditions much higher concentrations of UK63052 are required (micromolar). In contrast to luzopeptin UK63052 does not affect DNA mobility on denaturing gels. It seems that UK63052 binds reversibly more weakly than luzopeptin, yet stronger than echinomycin (which has no effect on DNA mobility) and alters DNA mobility by virtue of its effects on DNA structure. Since drug binding must extend the DNA helix by an equivalent of two base pairs the repeated blocks of adenines present in kinetoplast DNA will no longer be in phase and need not result in any overall curvature of the DNA molecule.

\section{Sequence Selectivity}

UK63052 displays the same selectivity for CpG as echinomycin although its ability to bind to such sites seems more sensitive to the nature of the surrounding sequences. In this respect UK63052 more closely resembles 3-amino triostin A which produces footprints on tyrT DNA at positions 20, 58 and $108^{12)}$ with other sites less favoured or unaffected. It seems that UK63052 prefers sequences of the type 
Fig. 6. Patterns of DEPC mediated DNA strand cleavage in the presence and absence of UK63052 and echinomycin.

(A)

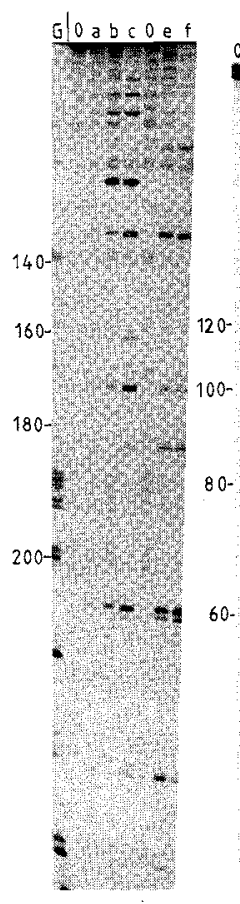

(B)

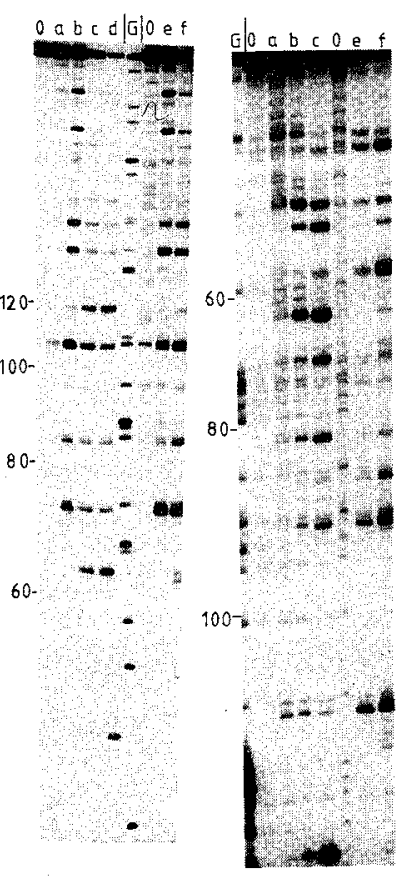

(D)

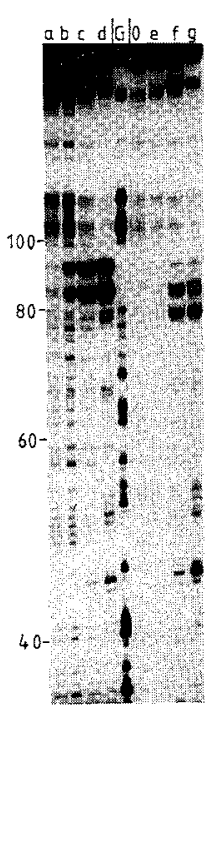

(A) Kinetoplast DNA labelled at the $3^{\prime}$-end of the bottom strand, (B) kinetoplast DNA labelled at the $3^{\prime}$-end of the top strand, (C) tyrT DNA labelled at the $3^{\prime}$-end of the top strand, (D) tyrT DNA labelled at the $3^{\prime}$-end of the bottom strand. The numbers correspond to the sequences shown in Fig. 2. Tracks labelled " $G$ " correspond to dimethylsulfate-piperidine markers specific for guanine. a) UK63052 $1 \mu \mathrm{M}$, b) UK63052 $5 \mu \mathrm{M}$, c) UK63052 $25 \mu \mathrm{M}$, d) UK63052 $100 \mu \mathrm{M}$, e) echinomycin $1 \mu \mathrm{M}, \mathrm{f}$ ) echinomycin $5 \mu \mathrm{M}$, g) echinomycin $25 \mu \mathrm{M}$.

(A/T)CG(A/T), though binding to other CG steps is not excluded. With kinetoplast DNA echinomycin binds less well to sequences GCG $(\mathrm{CGC})^{20}$, similar to its observed weaker binding to position 35 on tyrT DNA (GCGT) ${ }^{9}$; this preference seems to be exaggerated for UK63052.

What then is the origin of this modified sequence selectivity? The possible candidates include i) altering the chromophore from quinoxaline to quinoline, ii) substitution of the chromophore, iii) the precise nature of the cross-bridge, iv) the hydrophobic amino acid at the $N$-methyl valine position. The first possibility can be discounted since $1 \mathrm{QN}$ and $2 \mathrm{QN}$, quinoline analogues of echinomycin, produce identical footprints to the parent compound ${ }^{12)}$, although subtle differences in their equilibrium binding isotherms were detected $^{11)}$. The cross-bridge is unlikely to affect binding since it is the same length as echinomycin and the added substituents lie on the back of the drug molecule, facing away from the DNA helix. Crystal structures of echinomycin and triostin A complexed to oligonucleotide fragments reveal few van der Waals contacts between valine side chains and the sugar-phosphate backbone ${ }^{26)}$, consistent with the variable nature of the amino acid in this position. In addition quinomycin $\mathrm{C}$, which possess $N$-methyl-allo-isoleucine in this position, displays identical binding properties to echinomycin ${ }^{7}$. It therefore seems that amino acid substitution does not account for the modified selectivity. The most likely cause of the changes lies in the hydroxy substitution of the ring system. This will alter the charge distribution on the chromophores, thereby affecting the base pair stacking properties.

\section{Structural Changes}

The enhancements in DNAase I cleavage and the modification by DEPC confirm that UK63052 and echinomycin have different effects on DNA structure. The most likely explanation for this is that they 
induce different unwinding angles. Since enhancements generally seem to be more sensitive to weaker binding sites we can not rule out the possibility that the differences merely reflect changes in the nature of the secondary (weaker) binding sites, as well as the strong binding regions.

\section{Acknowledgements}

This work was supported by grants from the Medical Research Council, the Wellcome Trust and the Cancer Research Campaign. KRF is a Lister Institute Research Fellow.

\section{References}

1) Katagiri, K.; T. Yoshida \& K. Sato: Quinoxaline antibiotics. In Antibiotics. Mechanism of Action of Antimicrobial and Antitumour Agents. Eds., J. W. CoRCORAN \& F. E. HAHN, Vol. III, pp. 234 251, Springer, 1975

2) Dell, A.; D. H. Williams, H. R. Morris, G. A. Smith, J. Feeney \& G. C. K. Roberts: Structure revision of antibiotic echinomycin. J. Am. Chem. Soc. 97: 2497 2502, 1975

3) Kuroya, M.; N. Ishida, K. Katagiri, J. Shōiı, T. Yoshida, M. Mayama, K. Satō, S. MatsuUra, Y. Ninome \& O. SHIRATORI: Studies on quinoxaline antibiotics. I. General properties and the producing strains. J. Antibiotics, Ser. A 14: 324 329, 1961

4) WARING, M. J. \& L. P. G. WAKelin: Echinomycin: A bifunctional intercalating antibiotic. Nature 252: 653 657,1974

5) WARING, M. J. \& K. R. Fox: Molecular aspects of the interaction between quinoxaline antibiotics and nucleic acids. In Molecular Aspects of Anti-cancer Drug Action. Eds., S. NeIdLE \& M. J. WARING, pp. 127 156, Macmillan, 1983

6) WAKELIN, L. P. G. \& M. J. WARING: Binding of echinomycin to deoxyribonucleic acid. Biochem. J. 157: 721 740, 1976

7) LEE, J. S. \& M. J. WARING: Bifunctional intercalation and sequence specificity in the binding of quinomycin and triostin antibiotics to deoxyribonucleic acid. Biochem. J. 173: 115 128, 1978

8) VAN Dyke, M. W. \& P. B. DeRvan: Echinomycin binding sites on DNA. Science 225: 1122 1127, 1984

9) Low, C. M. L.; H. R. DREW \& M. J. WaRING: Sequence-specific binding of echinomycin to DNA: Evidence for conformational changes affecting flanking sequences. Nucleic. Acids Res. 12: 4865 4879, 1984

10) Low, C. M. L.; R. K. OLSEN \& M. J. WARING: Sequence preferences in the binding to DNA of triostin A and TANDEM as reported by DNase I footprinting. FEBS Lett. 176: 414 419, 1984

11) Fox, K. R.; D. Gauvreau, D. G. Goodwin, \& M. J. Waring: Binding of quinoline analogues of echinomycin to deoxyribonucleic acid: Role of the chromophores. Biochem. J. 191: 729 742, 1980

12) Low, C. M. L.; K. R. Fox \& M. J. WARING: DNA sequence selectivity of three biosynthetic analogues of the quinoxaline antibiotics. Anti-Cancer Drug Design 1: 149 160, 1986

13) Konishi, M.; H. Ohkuma, F. Sakai, T. Tsuno, H. Koshiyama, T. Naito \& H. Kawaguchi: Structures of BBM-928A, B, and C. Novel antitumor antibiotics from Actinomadura luzonensis. J. Am. Chem. Soc. 103: $1241 \sim 1243,1981$

14) Fox, K. R.; H. Davies, G. R. Adams, J. Portugal \& M. J. Waring: Sequence-specific binding of luzopeptin to DNA. Nucleic Acids Res. 16: 2489 2507, 1988

15) Searle, M. S.; J. G. Hall, W. A. Denny \& L. P. G. Wakelin: Interaction of the antitumour antibiotic luzopeptin with the hexanucleotide duplex d(5'GCATGC)2; one and two-dimensional NMR studies. Biochem. J. 259: $433 \sim 441,1989$

16) Rance, M. J.; J. C. Ruddock, M. S. Pacey, W. P. Cullen, L. H. Huang, M. T. Jefrerson, E. B. Whipple, H. MAEDA \& J. TONE: UK-63,052 complex, new quinomycin antibiotics from Streptomyces braegensis subsp. japonicus; taxonomy, fermentation, isolation, characterisation and antimicrobial activity. J. Antibiotics 42: 206 217,1989

17) Drew, H. R. \& A. A. TRAvers: DNA structural variations in the $E$. coli tyrT promoter. Cell 37: 491 502, 1984

18) Kitchin, P. A.; V. A. Klein, K. A. Ryan, K. L. GanN, C. A. Rauch, D. S. Kang, R. D. Wells \& P. T. ENGLUND: A highly bent fragment of Crithidia fasciculata kinetoplast DNA. J. Biol. Chem. 261: 11302 11309, 1986

19) Fox, K. R. \& M. J. WARING: DNA structural variations produced by actinomycin and distamycin as revealed by DNAase I footprinting. Nucleic Acids Res. 12: 9271 9285, 1984

20) Fox, K. R. \& E. KeNTEBE: Footprinting studies on the effect of echinomycin on the structure of a bent DNA fragment. Biochem. J. 269: 217 221, 1990

21) Portugal, J.; K. R. Fox, M. J. MCLeAN, J. L. RichenBerg \& M. J. Waring: Diethyl pyrocarbonate can detect 
a modified DNA structure induced by the binding of quinoxaline antibiotics. Nucleic Acids Res. 16: $3655 \sim 3670$, 1988

22) MCLean, M. J. \& M. J. WaRING: Chemical Probes reveal no evidence of Hoogsteen base pairing in complexes formed between echinomycin and DNA in solution. J. Mol. Recog. 1: 138 151, 1988

23) JEPPESEN, C. \& P. E. NIELSEN: Detection of intercalation-induced changes in DNA structure by reaction with diethylpyrocarbonate or potassium permanganate. Evidence against the induction of Hoogsteen base pairing by echinomycin. FEBS Lett. 231: 172 176, 1988

24) Mendel, D. \& P. B. DeRvan: Hoogsteen base pairs proximal and distal to echinomycin binding sites on DNA. Proc. Natl. Acad. Sci. U.S.A. 84: $910 \sim 914,1987$

25) Fox, K. R. \& C. Woolley: The strong binding of luzopeptin to DNA. Biochem. Pharm. 39: $941 \sim 948,1990$

26) Ughetto, G.; A. H. J. Wang, G. J. Quigley, G. van Der Marel, J. H. van Boom \& A. Rich: A comparison of the structure of echinomycin and triostin complexes to a DNA fragment. Nucleic Acids Res. 13: 2305 2323, 1985 